\title{
A Mixed-Attribute Approach in Ant-Miner Classification Rule Discovery Algorithm
}

\author{
Ayah Helal \\ School of Computing \\ University of Kent \\ Chatham Maritime, UK \\ amh58@kent.ac.uk
}

\author{
Fernando E. B. Otero \\ School of Computing \\ University of Kent \\ Chatham Maritime, UK \\ F.E.B.Otero@kent.ac.uk
}

\begin{abstract}
In this paper, we introduce Ant-Miner mA to tackle mixedattribute classification problems. Most classification problems involve continuous, ordinal and categorical attributes. The majority of Ant Colony Optimization (ACO) classification algorithms have the limitation of being able to handle categorical attributes only, with few exceptions that use a discretisation procedure when handling continuous attributes either in a preprocessing stage or during the rule creation. Using a solution archive as a pheromone model, inspired by the ACO for mixed-variable optimization $\left(\mathrm{ACO}_{\mathrm{MV}}\right)$, we eliminate the need for a discretisation procedure and attributes can be treated directly as continuous, ordinal, or categorical. We compared the proposed Ant-Miner ${ }_{\text {MA }}$ against $c$ Ant-Miner, an ACO-based classification algorithm that uses a discretisation procedure in the rule construction process. Our results show that Ant-Miner ma achieved significant improvements on computational time due to the elimination of the discretisation procedure without affecting the predictive performance.
\end{abstract}

\section{CCS Concepts}

-Computing methodologies $\rightarrow$ Supervised learning by classification;

\section{Keywords}

ant colony optimization, Ant-Miner, data mining, classification, continuous attributes

\section{INTRODUCTION}

Data mining is the process of extracting knowledge and patterns from data $[1,22]$. One of the major data mining areas is classification. Classification is concerned in finding patterns in data sets, then use those patterns to classify any new (future) data. Classification problems can be viewed as optimization problems, where the aim is to create the best model that represent the predictive patterns in

Permission to make digital or hard copies of all or part of this work for personal or classroom use is granted without fee provided that copies are not made or distributed for profit or commercial advantage and that copies bear this notice and the full citation on the first page. Copyrights for components of this work owned by others than the author(s) must be honored. Abstracting with credit is permitted. To copy otherwise, or republish, to post on servers or to redistribute to lists, requires prior specific permission and/or a fee. Request permissions from permissions@acm.org.

GECCO '16, July 20 - 24, 2016, Denver, CO, USA

(C) 2016 Copyright held by the owner/author(s). Publication rights licensed to ACM ISBN 978-1-4503-4206-3/16/07 . \$ \$15.00

DOI: http://dx.doi.org/10.1145/2908812.2908900 the data. The discovered classification model is then used to classify - predict the class attribute value of - new examples (unseen during training) based on the values of their predictor attributes. Using classification rules gives a more comprehensible classification model compared to black-box models such as SVMs or artificial neural networks [17], which are more difficult to be interpreted.

The first Ant Colony Optimization (ACO) classification algorithm, called Ant-Miner, was proposed in [16]. AntMiner and its extensions [7] were used to successfully extract IF-THEN classification rules from data. Each ant traverses a construction graph, where each node in the graph consists of a condition that the ant might choose to add to its rule. The majority of ACO-based classification algorithms are limited to cope only with categorical attributes, therefore continuous attributes must be discretised in a pre-processing stage. $c$ Ant-Miner $[13,14]$ was the first Ant-Miner extension to cope with continuous attributes directly. It employs a dynamic discretisation procedure during the rule construction: when an ant select a node representing a continuous attribute, the discretisation procedure is used to determine cutoff value in a dynamic manner. A potential drawback of this approach is its computational time: discretisation requires sorting and the evaluation of multiple candidate cuttoff values, which can significantly increase the computational time of the algorithm when applied to large data sets.

In this paper we propose a new approach to extract $I F$ THEN of classification rules, based on the ACO for mixedvariable optimization $\left(\mathrm{ACO}_{\mathrm{MV}}\right)$ [3]. Our approach handles the mixed attributes directly: attributes are categorized as continuous, ordinal and categorical attributes. This takes full advantage of the mechanisms of $\mathrm{ACO}_{\mathrm{MV}}$ to tackle mixed-variable optimization to cope with mixed-attribute classification problems. More importantly, it eliminates the need for a discretisation procedure, which improves the computational time for most of the large datasets. We compared the predictive accuracy and computational time performance of the proposed algorithm against $c$ Ant-Miner in 30 publicly available data sets. Our results show that the proposed algorithm significantly improves the computational time when compared to $c$ Ant-Miner while maintaining the predictive accuracy.

The remainder of this paper is organized as follows. We begin by reviewing the literature of ACO-based classification algorithms in Section 2. Then, we present an overview of $c$ Ant-Miner and $\mathrm{ACO}_{\mathrm{MV}}$ in Section 3. We then present our proposed Ant-Miner ${ }_{\text {MA }}$ algorithm in Section 4, com- 
putational results are presented in Section 5, and finally, conclusions and directions for future work are discussed in Section 6 .

\section{RELATED WORK}

There are two main approaches to apply ant colony optimization to create classification rules: grammar- and graphbased approaches. In grammar-based approaches, the rule creation is guided by a context-free grammar, which determines the valid structure of rules. The Grammar-Based Ant Programming (GBAP) algorithm $[9,10]$ was the first implementation of a grammar-based approach. Similar to most of ACO-based classification algorithms, GBAP does not cope with continuous attributes directly and it uses a discretisation procedure in a preprocessing stage.

Graph-based approaches started with Ant-Miner [16], which was limited to discrete datasets only. Several extensions of Ant-Miner have been proposed [7]. Ant-Miner2 [6] and Ant-Miner3 [5] presented a simple heuristic function using density estimation. Ant-Miner+ [8] extended Ant-Miner in several aspects: it uses a class based heuristic, since an ant pre-selects the predicted class value and extracts a rule accordingly; it also employs a different pheromone initialization and update procedure based on $\mathcal{M} \mathcal{A X}-\mathcal{M I N}$ ant system $(\mathcal{M M A S})[21]$, where the use of the lower and upper bound values allows the algorithm to avoid early stagnation of the search; and the complexity of the construction graph is reduced, in terms of the number of edges connecting vertices, by defining it as a direct acyclic graph (DAG).

Additionally, Ant-Miner+ employs a distinctive procedure for categorical and ordinal attributes. Categorical attributes have unordered nominal values (e.g., male and female), which were treated as a tuple (attribute, $=$,value). Ordinal attributes have a natural order (e.g., poor $<$ acceptable $<$ good), where the algorithm creates upper and lower bounds on the values chosen by the ant: the first type represents a lower bound of the interval and takes the form (attribute, $\leq$, value $_{i}$ ); the second type represents an upper bound of the interval and takes the form (attribute, $\geq$, value ${ }_{j}$ ), where value $_{i}$ and value $_{j}$ are values from the attribute domain. Continuous attributes are discretised in a pre-processing stage and then treated as ordinal attributes.

$c$ Ant-Miner was proposed in [13], where an entropy-based method is proposed for handling the continuous attributes discretisation during the rule construction process. The use of the minimum description length (MDL) principle in $c$ AntMiner to allow construction of discrete intervals with lower and upper bounds was proposed in [14]. Further improvements in the $c$ Ant-Miner are found in [18], where the authors proposed the use of multiple pheromone levels to extract rules predicting different class values. The latest improvements based on $c$ Ant-Miner were presented in [15], where using a new sequential covering strategy, each ant creates and evaluates a rule list (considering interactions among rules) rather than creating and evaluating a single rule like most versions of Ant-Miner. More recently, an extension to discover unordered rules (set of rules) instead of ordered rules (list of rules), with the aim of improving the interpretability of the discovered rules, was proposed in $[11,12]$.

Despite the Ant-Miner extensions proposed in the literature, extending Ant-Miner to use a archive-based pheromone model to handle continuous and categorical values (i.e., using $\mathrm{ACO}_{\mathrm{MV}}$ model ) is a research topic that has not been explored, to the best of our knowledge. Most of the proposed extensions handle continuous attributes in a pre-processing stage, while $c$ Ant-Miner employs a dynamic discretisation procedure using a heuristic to define intervals.

\section{BACKGROUND}

\section{1 $c$ Ant-Miner}

$c$ Ant-Miner uses a graph-based approach to extract $I F$ THEN classification rules from data. Let $r$ be a rule, each rule is a $n$-dimensional vector of terms $t$ that are joined with $A N D$, such that IF $t_{1}$ AND $t_{2} \ldots$... AND $t_{n}$ THEN (class), each term $t_{i}$ consist of a tuple (attribute, operator, value).

The $c$ Ant-Miner construction graph consists of a fully connected graph. Let $a_{i}$ be a nominal attribute and $v_{j i}$ be the $j$-th value of $a_{i}$ attribute. For $j=1, \ldots, b_{i}$, where $b_{i}$ is the number of values of attribute $a_{i}$, each $v_{i j}$ is added as a node $\left(a_{i},=, v_{i j}\right)$ to the graph. Let $c_{i}$ be a continuous attribute, only one node is added with $\left(c_{i}\right)$ to the graph - the operator and value are not defined for a continuous attribute node.

Suppose an ant $x$ is generating a rule $r_{x}$. It starts with an empty rule at node $i$ and probabilistically chooses to visit a node $j$ based on the amount of pheromone and heuristic information on the edge $E_{i j}$, given by

$$
P\left(E_{i j}\right)=\frac{\tau_{i j}^{\alpha} \cdot \eta_{j}^{\beta}}{\sum_{l \in \text { allowed i }} \tau_{i l}^{\alpha} \cdot \eta_{l}^{\beta}}
$$

where $\tau_{i j}$ is the pheromone value of the edge connecting node $i$ to node $j ; \eta_{j}$ is the value of the heuristic information for node $j$; node $l$ is a node in the neighbourhood of node $i$; the exponents $\alpha$ and $\beta$ are used to control the influence of the pheromone and heuristic information, respectively.

If a node with a nominal attribute is selected, then a term in the form $\left(a_{i}=v_{i j}\right)$ is added to the rule. If a node with a continuous attribute is selected, then a dynamic discretisation procedure based on the entropy measure is used to choose an operator and value to create a term in the form $\left(a_{i} \leq v_{i j}\right)$ or $\left(a_{i}>v_{i j}\right)$. This is done with a complexity of $O(n \log n)$, where $n$ is the number of the training instances, since the values need to be sorted and multiple cutoff values are evaluated.

An ant keeps adding terms until the rule covers less training examples than a user specified threshold, or all attributes are already added to the rule.

\section{2 $\quad \mathrm{ACO}_{\mathrm{MV}}$}

$\mathrm{ACO}_{\mathrm{MV}}$ [3] is applied to mixed variables optimization problems with $r$ real-valued variables, $c$ categorical-valued variables and $o$ ordinal-valued variables. The $\mathrm{ACO}_{\mathrm{Mv}}$ uses a solution archive (SA) as a form of pheromone model, instead of a pheromone matrix. The archive structure contains $R$ previously generated solutions. Each solution $S_{j}$ in the archive, for $j=\{1,2, \ldots, R\}$, is a vector containing $n$ dimensional real-valued components, $m$-dimensional indexvalued components and $o$-dimensional ordinal-valued components. The archive is sorted by the quality $Q$ of solutions, so that $Q\left(S_{1}\right) \geq Q\left(S_{2}\right) \geq \cdots \geq Q\left(S_{R}\right)$.

Each solution $S_{j}$ is associated with weight $w_{j}$ that is related to $Q\left(S_{j}\right)$, where $w_{j}$ is calculated using the Gaussian function given by 


$$
w_{j}=\frac{1}{q R \sqrt{2 \pi}} e^{\frac{-(\operatorname{ran} k(j)-1)^{2}}{2 q^{2} R^{2}}}
$$

where $q$ is a variable that is used to control the extend of the top-ranked solution influence on the construction of new solutions. The weight of solution $S_{j}$ is used during the creation of new solutions, as an indicator for the level of attractiveness of this solution. The higher the weight of the solution $S_{j}$, the higher the probability of sampling a new solution around the values of $S_{j}$.

$\mathrm{ACO}_{\mathrm{MV}}$ starts by randomly generating $R$ solutions in the archive. The solution construction phase starts by each ant $i$ generating a new $S_{i}$ candidate solution. When constructing solution $S_{i}$, a probabilistic solution construction method is used to sample new values from the solution archive according to each attribute type. At the end of an iteration, all solutions created by the ants in the colony are added to the archive. The archive is sorted and only the best $R$ solutions are kept and the remaining solutions are removed.

\subsubsection{Continuous variables}

Continuous variables are handled by $\mathrm{ACO}_{\mathrm{MV}}$ using $\mathrm{ACO}_{\mathbb{R}}$ [19], where each ant $i$ probabilistically chooses one solution from the archive based on

$$
P_{j}=\frac{w_{j}}{\sum_{l=1}^{R} w_{l}}
$$

where $P_{j}$ is the probability of selecting the $j$-th solution from the archive to sample the new continuous variable value around it, $R$ is the size of the archive, and $w_{j}$ is the weight associated with the $j$-th solution in the archive. Let $S_{i}$ denote a new solution sampled by ant $i$ around the chosen solution $S_{j}$ for continuous attribute $a$, the Gaussian probability density function (PDF) is given by

$$
\begin{gathered}
S_{i, a} \sim N\left(S_{j, a}, \sigma_{j, a}\right) \\
\sigma_{j, a}=\xi \sum_{r=1, j \neq r}^{R} \frac{\left|S_{j, a}-S_{r, a}\right|}{R-1}
\end{gathered}
$$

where $S_{j, a}$ is the value of the variable $a$ in the solution $j$ of the archive, $\sigma_{a, j}$ is the average distance between the value of the variable $a$ in the solution $j$ and the value of $a$ in all the other solutions in the archive and $\xi$ is a user-defined value representing the convergence speed of the algorithm.

\subsubsection{Ordinal variables}

Ordinal variables are variables whose order have a meaning, e.g., small < medium < large. $\mathrm{ACO}_{\mathrm{MV}}$ handles ordinal variables as continuous variables, where the continuous value is the index of the chosen value in the ordered attribute values. Then, a final step is to round up the value generated from Equation (4) to the nearest index. Using this relaxed continuous sampling allows the algorithm to take in consideration the order of the attribute values.

\subsubsection{Categorical variables}

Categorical variables are treated differently by $\mathrm{ACO}_{\mathbf{M V}}$. Suppose a categorical variable $i$ that has $t$ possible values, each ant has to choose $v_{l}$ where $v_{l}^{i} \in\left\{v_{1}^{i}, v_{1}, . ., v_{t}^{i}\right\}$. The probability $P_{l}^{i}$ to choose $v_{l}$ is given by

$$
P_{l}^{i}=\frac{\alpha_{l}}{\sum_{l=1}^{t} \alpha_{j}}
$$

where the variable $\alpha_{l}$ is weight function for each value of the categorical variable, based on the weight of the solution, calculated as

$$
\alpha_{l}= \begin{cases}\frac{w_{j i}}{u_{j}}+\frac{q}{\kappa}, & \text { if } \kappa>0, u_{j}>0, \\ \frac{w_{j i}}{u_{j}}, & \text { if } \kappa=0, u_{j}>0, \\ \frac{q}{\kappa}, & \text { if } \kappa>0, u_{j}=0,\end{cases}
$$

where $\alpha_{l}$ represents the weight associated with $v_{l}, w_{j i}$ is the weight of the first solution that uses the value $v_{j}$ in the archive, $u_{j}$ is the number of solutions that use the value $v_{j}$ in the archive, $\kappa$ is the number of values in this attribute that are not used in the archive, and $q$ is a variable that is used to control the extend of the top-ranked solution influence on the construction of new solutions.

The categorical sampling procedure allow an ant to consider two components when sampling a new value. The first component biases the sampling towards values that are used in high-quality solutions but do not occur very frequently in the archive. The second component biases the sampling towards unexplored values in that attribute.

\section{MIXED-ATTRIBUTES IN ANT-MINER}

The proposed algorithm Ant-Miner $\mathbf{M A}$ uses an $\mathrm{ACO}_{\mathbf{M V}}$ procedure to handle mixed attributes types, eliminating the need of an entropy-based discretisation when handling a continuous attribute, and also coping with ordinal attributes. The archive is used to sample conditions for the creation of the rules instead of traversing a construction graph. A highlevel pseudocode of Ant-Minerma is shown in Algorithm 1.

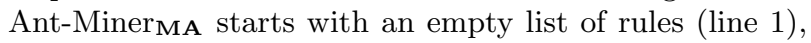
and iteratively (outer while loop in line 2) adds the best rule found along the iterative process to the list of rules (line 20), while the number of uncovered training examples is greater than a maximum uncovered value in a sequential covering fashion.

At each iteration, a single rule is created by an $\mathrm{ACO}_{\mathrm{MV}}$ procedure (lines $3-18$ ). It starts by initializing the archive with $R$ random generated rules (line 3 ). Then, each ant generates a new rule (lines 6-11). Once $m$ new rules have been generated, where $m$ is the number of ants, they are added into the solution archive (line 12). The $R$ and $m$ rules are sorted and the $m$ worst ones are removed from the archive. The procedure to create a new rule is repeated until the maximum number of iterations has been reached, or a restart procedure is once applied.

\subsection{Archive and Rule Structure}

As aforementioned, the archive consist of $R$ rules. Each rule consist of a vector of $n$-dimensional terms, where $n$ is the number of attributes in the dataset. Each term $t_{j}$ in rule $R_{i}$ contains a flag to indicate if this term is enabled or not, an operator and value(s). For continuous attributes, the operator could be either LESS THAN OR EQUAL, GREATER THAN or IN RANGE; categorical attributes' operator is always 


\begin{tabular}{|c|c|c|c|c|c|c|c|c|c|c|c|c|c|c|c|c|c|c|}
\hline & \multicolumn{4}{|c|}{ Continuous attribute $\left(A_{r}\right)$} & & & \multicolumn{3}{|c|}{ Categorical attribute $\left(A_{c}\right)$} & & & \multicolumn{3}{|c|}{ Ordinal attribute $\left(A_{o}\right)$} & \multirow[b]{2}{*}{$\ldots$} & \multirow[b]{2}{*}{$\ldots$} & \multirow[b]{2}{*}{$\mathrm{f}(S)$} & \multirow[b]{2}{*}{$w$} \\
\hline & Flag & Op & Value1 & Value2 & $\ldots$ & $\ldots$ & Flag & Op & Value & $\ldots$ & $\ldots$ & Flag & $\mathrm{Op}$ & Value & & & & \\
\hline$S_{1}$ & $\mathrm{~T}$ & $>$ & $v_{1}$ & - & $1 \ldots$ & $\ldots$ & $\mathrm{T}$ & $=$ & $v_{2}$ & $\mid \ldots$ & $\ldots$ & $\mathrm{F}$ & -1 & - & $\mid \ldots$ & $\ldots$ & $\mathrm{f}\left(S_{1}\right)$ & $w_{1}$ \\
\hline$S_{2}$ & $\mathrm{~T}$ & InR & $v_{3}$ & $v_{4}$ & $\mid \ldots$ & $\ldots$ & $\mathrm{F}$ & - & - & $\mid \ldots$ & $\ldots$ & $\mathrm{T}$ & $\leq 1$ & $v_{5}$ & $\mid \ldots$ & $\ldots$ & $\mathrm{f}\left(S_{2}\right)$ & $w_{2}$ \\
\hline$S_{3}$ & $\mathrm{~F}$ & - & - & - & $\mid \ldots$ & $\ldots$ & $\mathrm{T}$ & $=$ & $v_{6}$ & $\ldots$ & $\ldots$ & $\mathrm{T}$ & $\geq 1$ & $v_{7}$ & $\ldots$ & $\ldots$ & $\mathrm{f}\left(S_{3}\right)$ & $w_{3}$ \\
\hline $\begin{array}{l}\dot{ } \\
\dot{\bullet} \\
\dot{\bullet}\end{array}$ & & & • & $\because$ & & & & & • & & & & & •. & & & $\begin{array}{l}\dot{ } \\
\dot{ } \\
\dot{ } \\
\dot{ }\end{array}$ & $\begin{array}{l}\dot{ } \\
\dot{ } \\
\dot{ }\end{array}$ \\
\hline$S_{R}$ & $\mathrm{~T}$ & $\leq$ & $v_{8}$ & - & $\mid \ldots$ & $\ldots$ & $\mathrm{F}$ & - & - & $\mid \ldots$ & $\ldots$ & $\mathrm{T}$ & $\leq 1$ & $v_{9}$ & $1 \ldots$ & $\ldots$ & $\mathrm{f}\left(S_{R}\right)$ & $w_{R}$ \\
\hline
\end{tabular}

Figure 1: Archive Structure: example of 3 solutions of the archive, each solution showing a single example of each attribute type. $A_{r}$ is a real-valued (continuous) attribute, $A_{c}$ is a categorical attribute and $A_{o}$ is an ordinal attribute.

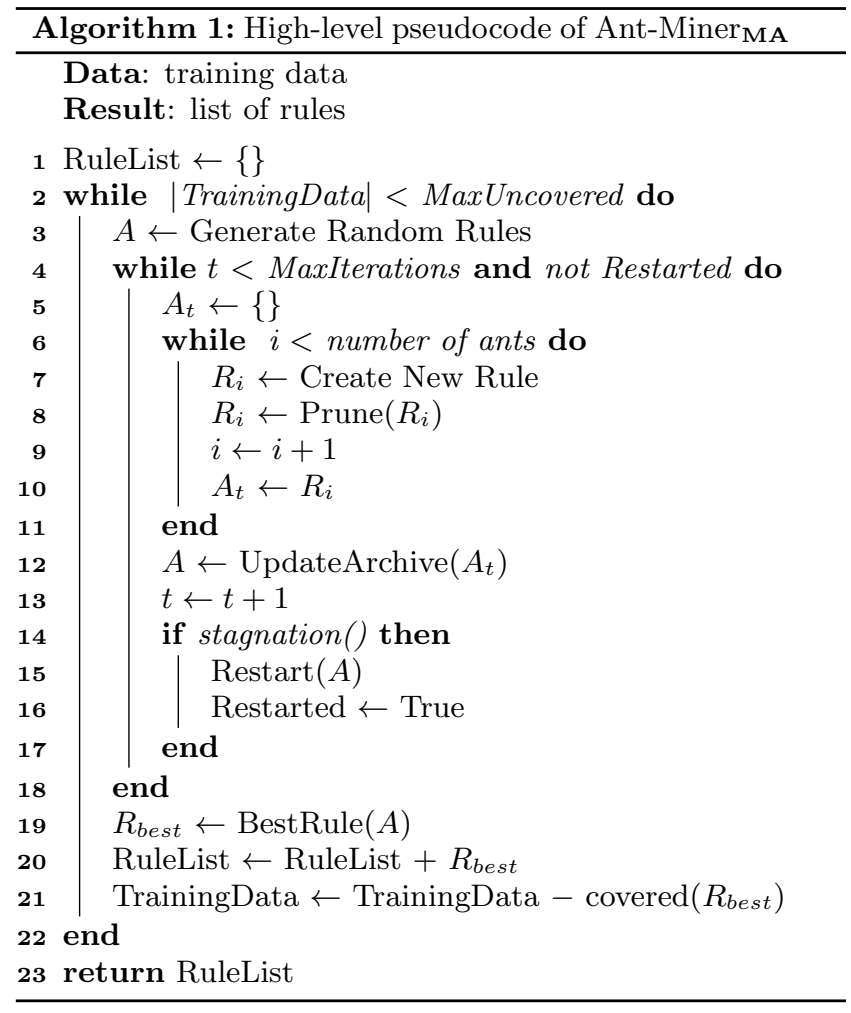

EQUAL; and ordinal attributes have an operator of either LESS THAN OR EQUAL Or GREATER THAN OR EQUAL.

Figure 1 illustrates a solution archive with 3 solutions, each solution showing a single example of each attribute type. The solutions are stored according to their quality in the archive, where the best is stored at the top (highest ranking) and the worse at the bottom (worst ranking). The archive stores the quality of each rule in $f(S)$ and the weight in $w$ calculated by Equation (2).

In Figure 1, $A_{r}$ is an example of a real-valued (continuous) attribute in the dataset, $A_{c}$ is an example of a categorical attribute in the dataset and $A_{o}$ is an example of an ordinal attribute in the dataset. The solution $S_{1}$ is an example in which the continuous attribute $A_{r}$ is enabled (Flag $=\mathrm{T}$ ), the operator is GREATER THAN and value $v_{1}$ is set-representing the term $A_{r}>v_{1}$. The categorical attribute $A_{c}$ is enabled ( $\mathrm{Flag}=\mathrm{T}$ ), the default operator is EQUAL and value $v_{2}$ is set-representing the term $A_{c}=v_{2}$. The ordinal attribute $A_{o}$ is not enabled (Flag $=$ F) and so it has no values for operator and value.

The solution $S_{2}$ is an example in which the continuous attribute $A_{r}$ is enabled $(\mathrm{Flag}=\mathrm{T})$, the operator is IN RANGE and two values $v_{3}$ and $v_{4}$ are set-representing the term $v_{3} \leq A_{r}<v_{4}$. The categorical attribute $A_{c}$ is not enabled showing $(\mathrm{Flag}=\mathrm{F})$ and so it has no values for operator and value. The ordinal attribute $A_{o}$ is enabled ( $\mathrm{Flag}=\mathrm{T}$ ), the operator is LESS THAN OR EQUAL and value $v_{5}$ is setrepresenting the term $A_{o} \leq v_{5}$. Solution $S_{3}$ follows a similar representation.

\subsection{Archive Initialization}

In the archive initialization procedure, each rule is randomly initialized. Rule initialization starts with an unbiased random probability to enable each the term, then according to its type it continues the rule initialization. If it is a continuous term, it uses an unbiased random probability to set the operator from the set \{LESS THAN OR EQUAL, GREATER THAN, IN RANGE\}. The value of the continuous attribute is generated using a random value from a normal distribution in the range of values of the attribute. In case of the IN RANGE operator, two values are generated and the values will only be accepted if they make the operator valid.

Ordinal terms also use an unbiased random probability to choose the operator from the set \{LESS THAN OR EQUAL, GREATER THAN OR EQUAL\}, then an unbiased random value for the index is generated. For categorical terms, a default EQUAL operator is added, then an unbiased random value for the index is generated.

After the random initialization of each rule, a rule is pruned to remove irrelevant terms enabled by the stochastic nature of the initialization. Then, if the number of of covered instances is greater or equal to the user-defined minimum limit, it will be added to the archive. Finally, the rules in the archive are ordered according to their quality, so that $f\left(S_{1}\right) \geq f\left(S_{2}\right) \geq \cdots \geq f\left(S_{R}\right)$. 


\subsection{Rule Creation}

Rule creation follow the following steps:

1. For each term, we start by considering the probability to include the term or not. The decision is handled as a categorical variable, since we are dealing with boolean \{TRUE, FALSE $\}$ values. We use the same procedure in Section 3.2.3 to probabilistic sample a boolean value.

2. If the term is enabled, we set the operator according to the attribute type. If the attribute type is categorical, we set the operator to EQUAL. If it is continuous, the decision is handled as a categorical attribute of three values \{LESS THAN OR EQUAL, GREATER THAN, IN RANGE\}, using the same procedure in Section 3.2.3 to probabilistic sample an operator based on the subset of the archive rules that have this term enabled. While for ordinal attributes, the decision is handled as a categorical attribute of two values LESS THAN OR EQUAL, GREATER THAN OR EQUAL\}, using the same procedure in Section 3.2.3 to probabilistic sample an operator based on the subset of the archive rules that have this term enabled.

3. The value of each attribute is then sampled. If the attribute type is continuous, we use the continuous sampling procedure in Section 3.2.1 based on the subset of the archive rules that have this term enabled and using the same operator. If the attribute is ordinal, we use the continuous sampling procedure in Section 3.2.2 to benefit from the order of the attribute, based on the subset of the archive rules that have this term enabled and using the same operator. While for categorical terms we use the discrete sampling procedure in Section 3.2.3 based on the subset of the archive terms that are enabled.

4. After the creation of each term, we apply the rule to the training set. If the rule covered less than the minimum instances, the term is disabled. If it covers more than the minimum instances, the term is added and the process is repeated for the next term, until all terms are considered.

\subsection{Rule Pruning}

Ant-Miner ${ }_{\text {MA }}$ applies different heuristics for the rule refinement and rule selection following a similar approach proposed in [20]. We use the $m$-estimate measure in the rule selection and archive ordering, given by

$$
Q=\frac{T P+m \cdot\left(\frac{P}{P+N}\right)}{T P+F P+m}
$$

where TP and FP are number of the correct classified and misclassified instances by the rule, respectively; $P$ and $N$ are the total number of instances that are in the positive and negative class in training dataset, respectively. ${ }^{1}$ The value $m=22.466$ used in our approach has been determined experimentally in [2] to be optimum value for the $m$-estimate measure.

For the pruning function, we use the sensitivity specificity function, as employed in Ant-Miner, given by

\footnotetext{
${ }^{1}$ An instance is considered negative if it is from a class different than the class predicted by the rule
}

Table 1: Parameters: Ant-Miner ${ }_{\text {MA }}$ uses the first three parameters in table, while remaining are used by both Ant-Miner ${ }_{\text {MA }}$ and $c$ Ant-Miner.

\begin{tabular}{lr}
\hline Parameters & Value \\
\hline$q$ & 0.025495 \\
$\xi$ & 0.6795 \\
$R$ & 90 \\
Minimum Covered & 10 \\
Max Uncovered & 10 \\
Max Iterations & 1500 \\
Number of Ants & 60 \\
Stagnation Test & 10 \\
\hline
\end{tabular}

$$
Q_{\text {Pruning }}=\frac{T P}{T P+F N} \cdot \frac{T N}{F P+T N}
$$

where $T P, F P, T N$ and $F N$ stand for true positives, false positives, true negatives and false negatives, respectively.

The pruning function starts by disabling the last term in the rule and if the quality of the rule does not decrease, it permanently removes the term; this process is repeated to disable the next one until disabling a term decreases the quality of the rule or the rule has only one term enabled. In this case, the pruning stops, returning the rule without removing the last term. This is the same pruning procedure used by $c$ Ant-Miner [14].

\subsection{Restart Strategy}

Ant-Miner MA uses a simple restart strategy to avoid stagnation. The restart strategy is triggered if the best rule of the current iteration is exactly the same as the best rule constructed by a user defined number of previous iterations, which works as stagnation test. When the restart strategy is triggered, all the rules in the archive are reinitialized except the best-so-far rule. The restart is performance only once.

\section{COMPUTATIONAL RESULTS}

Our computational results are calculated using 30 publicly available dataset from the UCI Machine Learning Repository [4], presented in Table 2. Ant-Miner ${ }_{\text {MA }}$ uses the first three parameters in Table 1 for the archive settings, while the rest of the parameters are used by both Ant-MinermA and $c$ Ant-Miner. The archive settings parameters were empirical chosen based on preliminary experiments. The remaining parameters were based on $c$ Ant-Miner default values [14]. The $c$ Ant-Miner implementation used in the experiments is $c$ Ant-Miner $2_{M D L}[14]^{2}$, which can create intervals with lower and upper bounds for continuous attributes.

We ran both algorithms for 15 times in a tenfold crossvalidation settings, the average results (over the 150 individual runs) are shown in Table 3. We ran Wilcoxon rank sum test on the 30 datasets to show if there are statistically significant differences in terms of both predictive accuracy and computational time. For a fair comparison, both algorithms are implemented in Java running on the same environment.

\footnotetext{
${ }^{2}$ We used the $c$ Ant-Miner $2_{M D L}$ variation available from https://github.com/febo/myra.
} 
Table 2: Summary of the datasets used in the experiments: datasets from 1 to 18 are considered small datasets, while the remaining ones are considered large datasets due to either the large number of attributes or the large number of instances.

\begin{tabular}{|c|c|c|c|c|c|c|c|}
\hline \multirow[b]{2}{*}{$\#$} & \multirow[b]{2}{*}{ Dataset } & \multirow[b]{2}{*}{ Size } & \multirow[b]{2}{*}{ \#Classes } & \multicolumn{4}{|c|}{ Attributes } \\
\hline & & & & Total & \#Ordinal & \#Categorical & \#Continuous \\
\hline 1 & breast-tissue & 106 & 6 & 9 & 0 & 0 & 9 \\
\hline 2 & iris & 150 & 3 & 4 & 0 & 0 & 4 \\
\hline 3 & wine & 178 & 3 & 13 & 0 & 0 & 13 \\
\hline 4 & parkinsons & 195 & 2 & 22 & 0 & 0 & 22 \\
\hline 5 & glass & 214 & 7 & 9 & 0 & 0 & 9 \\
\hline 6 & breast-1 & 286 & 2 & 9 & 4 & 5 & 0 \\
\hline 7 & heart-h & 294 & 5 & 13 & 3 & 3 & 7 \\
\hline 8 & heart-c & 303 & 5 & 13 & 3 & 3 & 7 \\
\hline 9 & liver-disorders & 345 & 2 & 6 & 0 & 0 & 6 \\
\hline 10 & ionosphere & 351 & 2 & 34 & 0 & 0 & 34 \\
\hline 11 & dermatology & 366 & 6 & 34 & 33 & 0 & 1 \\
\hline 12 & cylinder-bands & 540 & 2 & 35 & 2 & 14 & 19 \\
\hline 13 & breast-w & 569 & 2 & 30 & 0 & 0 & 30 \\
\hline 14 & balance-scale & 625 & 3 & 4 & 4 & 0 & 0 \\
\hline 15 & credit-a & 690 & 2 & 14 & 4 & 4 & 6 \\
\hline 16 & pima & 768 & 2 & 8 & 0 & 0 & 8 \\
\hline 17 & annealing & 898 & 6 & 38 & 0 & 29 & 9 \\
\hline 18 & credit-g & 1000 & 2 & 20 & 11 & 2 & 7 \\
\hline 19 & MiceProtein & 1080 & 8 & 80 & 0 & 3 & 77 \\
\hline 20 & HillValley & 1212 & 2 & 100 & 0 & 0 & 100 \\
\hline 21 & Magic & 19020 & 2 & 10 & 0 & 0 & 10 \\
\hline 22 & Nomao & 34465 & 2 & 118 & 0 & 29 & 89 \\
\hline 23 & bank-additional & 41188 & 2 & 20 & 0 & 10 & 10 \\
\hline 24 & $\mathrm{eb}$ & 45781 & 31 & 3 & 0 & 1 & 2 \\
\hline 25 & adult & 48842 & 2 & 14 & 0 & 8 & 6 \\
\hline 26 & connect 4 & 67557 & 3 & 42 & 0 & 42 & 0 \\
\hline 27 & diabetes & 101766 & 3 & 47 & 2 & 34 & 11 \\
\hline 28 & SkinNonSkin & 245057 & 2 & 3 & 0 & 0 & 3 \\
\hline 29 & ForestType & 581012 & 7 & 54 & 0 & 44 & 10 \\
\hline 30 & PokerHand & 1025010 & 10 & 10 & 5 & 0 & 5 \\
\hline
\end{tabular}

Table 3 shows that Ant-Miner ${ }_{\text {MA }}$ has a higher average rank of 1.43 for the predictive accuracy, where $c$ Ant-Miner has an average rank of 1.57 . In terms of computational time, Ant-Miner ${ }_{\text {MA }}$ has a rank of 1.10, while $c$ Ant-Miner has a rank of 1.87. Most of the datasets show an order of magnitude improvement of Ant-Minerma over $c$ Ant-Miner in terms of computational time. Considering both number of attributes and instances size, the largest datasets are forest type, poker hand and diabetes, respectively. Most notably, $c$ Ant-Miner running a single diabetes execution takes up to 3.5 days, while Ant-Miner ${ }_{\text {A }}$ takes just over 1 hour. AntMinermA would take 45 minutes for a single run in poker hand, while $c$ Ant-Miner almost 8 hours. These results show that the use of the solution archive as pheromone model does not affect the accuracy, while improving the computational time since the discretisation procedure can be eliminated.

Our approach seems to show a small limitation when the number of attributes increases over 50, where we could observe an improvement in computational time of only around $25 \%$ in forest type dataset. In cases where there is a large number of attributes but a smaller number of instanceswhich means that the discretisation overhead is less notice- able - such as in the datasets Nomao (119 attributes, 34465 instances), Hill Valley (101 attributes, 1212 instances) and Mice Protein (81 attributes, 1080 instances), Ant-MinermA running time increases in relation to $c$ Ant-Miner. We hypothesised that this is due to the use of heuristic information in cAnt-Miner, which allows the algorithm to quickly identify irrelevant attributes and not use them in the rule creation process.

Table 4 indicates that there is no statistically significantly differences between Ant-Miner ${ }_{\text {MA }}$ and $c$ Ant-Miner in term of predictive accuracy $(p=0.24200)$. In the case of computational time, Ant-Minerma improvement is statistically significant $(p=0.00036)$. Overall, we consider these results positive. The use of a rule creation process inspired by $\mathrm{ACO}_{\mathrm{MV}}$ led to a statistically significant computational time improvement in Ant-Miner MA compared to $c$ Ant-Miner, without affecting the predictive accuracy.

\section{CONCLUSION}

We introduced Ant-Minerma to tackle mixed-attribute classification problems, based on $\mathrm{ACO}_{\mathrm{MV}}$. The use of a solution archive allows the algorithm to deal with categor- 
Table 3: Average predictive accuracy and computational time (average [standard deviation]) of $c$ Ant-Miner and Ant-Miner ${ }_{\text {A }}$ over 15 runs of tenfold cross-validation. The last row of the table shows the average rank of the algorithm. The best value of a given dataset is shown in bold.

\begin{tabular}{|c|c|c|c|c|}
\hline \multirow[b]{2}{*}{ Dataset } & \multicolumn{2}{|c|}{ Accuracy } & \multicolumn{2}{|c|}{ Computational time (seconds) } \\
\hline & Ant-Miner MA & $c$ Ant-Miner & Ant-Miner MA & $c$ Ant-Miner \\
\hline breast-tissue & $60.24[0.97]$ & $64.24[0.24]$ & $0.38[0.01]$ & $0.67[0.02]$ \\
\hline iris & $93.6[0.31]$ & $94.27[0.11]$ & $0.28[0.00]$ & $0.49[0.00]$ \\
\hline wine & $90.78[0.40]$ & $93.52[0.07]$ & $0.33[0.01]$ & $0.56[0.04]$ \\
\hline parkinsons & $86.29[0.64]$ & $85.22[0.40]$ & $0.78[0.01]$ & $2.87[0.25]$ \\
\hline glass & $63.24[0.50]$ & $59.18[0.32]$ & $0.50[0.00]$ & $2.66[0.42]$ \\
\hline breast-l & $71.46[0.34]$ & $76.17[0.11]$ & $0.54[0.01]$ & $1.28[0.14]$ \\
\hline heart-h & $64.37[0.29]$ & $64.81[0.33]$ & $0.72[0.00]$ & $12.61[0.62]$ \\
\hline heart-c & $56.94[0.54]$ & $57.42[0.32]$ & $0.76[0.02]$ & $10.91[0.64]$ \\
\hline liver-disorders & $63.13[0.49]$ & $62.26[0.18]$ & $0.47[0.01]$ & $1.81[0.08]$ \\
\hline ionosphere & $89.28[0.37]$ & $88.84[0.25]$ & $1.27[0.03]$ & $5.97[0.65]$ \\
\hline dermatology & $89.42[0.47]$ & $89.02[0.25]$ & $1.31[0.02]$ & $16.67[1.49]$ \\
\hline cylinder-bands & $69.32[0.35]$ & $70.28[0.22]$ & $3.15[0.08]$ & $29.54[1.31]$ \\
\hline breast-w & $93.53[0.22]$ & $94.28[0.11]$ & $2.31[0.04]$ & $5.40[0.29]$ \\
\hline balance-scale & $80.10[0.22]$ & $68.34[0.08]$ & $0.50[0.01]$ & $5.95[0.38]$ \\
\hline credit-a & $85.19[0.22]$ & $85.74[0.11]$ & $1.10[0.01]$ & $11.57[0.79]$ \\
\hline pima & $75.30[0.21]$ & $67.45[0.07]$ & $0.93[0.01]$ & $3.69[0.42]$ \\
\hline annealing & $96.68[0.14]$ & $97.02[0.09]$ & $4.79[0.16]$ & $10.76[0.77]$ \\
\hline credit-g & $74.19[0.14]$ & $69.39[0.17]$ & $1.69[0.02]$ & $39.1[2.11]$ \\
\hline MiceProtein & $62.57[0.37]$ & $99.07[0.43]$ & $26.79[0.61]$ & $8.53[0.65]$ \\
\hline HillValley & $52.65[0.19]$ & $51.35[0.11]$ & $37.93[0.71]$ & $19.12[0.91]$ \\
\hline Magic & $82.67[0.05]$ & $70.41[0.01]$ & $25.56[0.61]$ & $155.07[2.01]$ \\
\hline Nomao & $87.56[0.05]$ & $90.66[0.02]$ & $2308.57[98.25]$ & $779.77[28.15]$ \\
\hline bank-additional & $89.49[0.02]$ & $89.87[0.01]$ & $185.27[2.49]$ & $1970.72[60.55]$ \\
\hline eb & $65.00[0.05]$ & $64.58[0.01]$ & $25.38[0.36]$ & $321.58[1.11]$ \\
\hline adult & $84.74[0.03]$ & $79.73[0.04]$ & $236.54[3.83]$ & 5048.44 [165.33] \\
\hline connect 4 & $68.18[0.02]$ & $67.83[0.01]$ & $1273.66[7.97]$ & $14380.04[535.72]$ \\
\hline diabetes & $55.83[0.09]$ & $54.23[0.13]$ & $4008.11[141.34]$ & * $388023.6[3580.67]$ \\
\hline SkinNonSkin & $98.91[0.02]$ & $97.54[0.00]$ & $125.41[1.56]$ & $1484.52[5.27]$ \\
\hline ForestType & $68.55[0.07]$ & $63.09[0.07]$ & $30649.92[695.20]$ & $53336.53[3946.86]$ \\
\hline PokerHand & $51.97[0.04]$ & $50.2[0.00]$ & $2577.19[43.07]$ & $27872.59[2286.18]$ \\
\hline Rank & 1.43 & 1.57 & 1.10 & 1.87 \\
\hline
\end{tabular}

* Result of a single tenfold execution due to high computational time.

Table 4: Wilcoxon rank sum tests (at the $\alpha=$ 0.05 level) on predictive accuracy and computational time. Statistically significant differences are shown in bold.

\begin{tabular}{lrrrcr}
\hline & Size & W + & W- & Z & $p$ \\
\hline Accuracy & 30 & 289.5 & 175.5 & -1.1724 & 0.24200 \\
Time & 30 & 59 & 406 & -3.5686 & $\mathbf{0 . 0 0 0 3 6}$ \\
\hline
\end{tabular}

ical, continuous and ordinal attributes directly, without required a discretisation procedure. The rule creation then uses $\mathrm{ACO}_{\mathrm{MV}}$ strategies to sample values for each attribute to create the antecedent of a rule.

We compared Ant-Miner $\mathrm{MA}$ against $c$ Ant-Miner using publicly available datasets, an ACO-based classification algorithm capable of dealing with continuous attributes using a dynamic discretisation procedure. Our results show that the proposed Ant-Miner mA statistically significantly improves the computational time of the algorithm with no negative effects on its accuracy - in most cases, an order of magnitude improvements were observed. This would enable AntMinerma to be applied to much larger datasets, mitigating the restriction on computational time.

There are several interesting directions for future research. First, it would be interesting to further investigate the effect of the number of attributes in the rule creation process. Our current results indicates that a large number of attributes might affect Ant-Minerma computational time. Second, $\mathrm{ACO}_{\mathrm{MV}}$ does not provide an explicit mechanism to incorporate heuristic information, which can be used to quickly identify irrelevant attributes. Therefore, incorporating heuristic information into Ant-Miner ${ }_{\text {MA }}$ can potentially improve its computational time by allowing the algorithm quickly identify irrelevant attributes. In addition, an extension to create a complete list of rules instead of one rule at a time is a research direction worth further exploration.

\section{REFERENCES}

[1] U. M. Fayyad, G. Piatetsky-Shapiro, and P. Smyth. 
Advances in knowledge discovery and data mining. chapter From Data Mining to Knowledge Discovery: An Overview, pages 1-34. American Association for Artificial Intelligence, Menlo Park, CA, USA, 1996.

[2] F. Janssen and J. Fürnkranz. On the quest for optimal rule learning heuristics. Machine Learning, 78(3):343-379, 2010.

[3] T. Liao, K. Socha, M. Montes de Oca, T. Stützle, and M. Dorigo. Ant colony optimization for mixed-variable optimization problems. IEEE Transactions on Evolutionary Computation, 18(4):503-518, 2014.

[4] M. Lichman. UCI Machine Learning Repository, 2013. Irvine, CA: University of California, School of Information and Computer Science. [http://archive.ics.uci.edu/ml].

[5] B. Liu, H. Abbas, and B. McKay. Classification rule discovery with ant colony optimization. In IEEE/WIC International Conference on Intelligent Agent Technology (IAT 2003), pages 83-88, Oct 2003.

[6] B. Liu, H. A. Abbass, and B. McKay. Density-Based Heuristic for Rule Discovery with Ant-Miner. In The 6th Australia-Japan Joint Workshop on Intelligent and Evolutionary System, pages 180-184, 2002.

[7] D. Martens, B. Baesens, and T. Fawcett. Editorial survey: swarm intelligence for data mining. Machine Learning, 82(1):1-42, 2011.

[8] D. Martens, M. De Backer, R. Haesen, J. Vanthienen, M. Snoeck, and B. Baesens. Classification with ant colony optimization. IEEE Transactions on Evolutionary Computation, 11(5):651-665, Oct 2007.

[9] J. Olmo, J. Romero, and S. Ventura. A grammar based ant programming algorithm for mining classification rules. In 2010 IEEE Congress on Evolutionary Computation (CEC), pages 1-8, July 2010.

[10] J. Olmo, J. Romero, and S. Ventura. Classification rule mining using ant programming guided by grammar with multiple pareto fronts. Soft Computing, 16(12):2143-2163, 2012.

[11] F. Otero and A. Freitas. Improving the Interpretability of Classification Rules Discovered by an Ant Colony Algorithm. In Genetic and Evolutionary Computation Conference (GECCO-2013), pages 73-80, New York, NY, USA, 2013. ACM Press.

[12] F. Otero and A. Freitas. Improving the Interpretability of Classification Rules Discovered by an Ant Colony
Algorithm: Extended Results. Evolutionary Computation (in press), pages 1-25, 2015.

[13] F. Otero, A. Freitas, and C. Johnson. cAnt-Miner: An Ant Colony Classification Algorithm to Cope with Continuous Attributes. In Ant Colony Optimization and Swarm Intelligence, volume 5217 of Lecture Notes in Computer Science, pages 48-59. Springer Berlin Heidelberg, 2008.

[14] F. Otero, A. Freitas, and C. Johnson. Handling continuous attributes in ant colony classification algorithms. In IEEE Symposium on Computational Intelligence and Data Mining (CIDM '09), pages 225-231, March 2009.

[15] F. Otero, A. Freitas, and C. Johnson. A new sequential covering strategy for inducing classification rules with ant colony algorithms. IEEE Transactions Evolutionary Computation, 17(1):64-76, 2013.

[16] R. Parpinelli, H. Lopes, and A. Freitas. Data mining with an ant colony optimization algorithm. IEEE Transactions on Evolutionary Computation, 6(4):321-332, Aug 2002.

[17] J. R. Quinlan. C4.5: Programs for Machine Learning. Morgan Kaufmann Publishers Inc., San Francisco, CA, USA, 1993.

[18] K. M. Salama, A. M. Abdelbar, F. E. Otero, and A. A. Freitas. Utilizing multiple pheromones in an ant-based algorithm for continuous-attribute classification rule discovery. Applied Soft Computing, 13(1):667-675, 2013.

[19] K. Socha and M. Dorigo. Ant colony optimization for continuous domains. European Journal of Operational Research, 185(3):1155-1173, 2008.

[20] J. Stecher, F. Janssen, and J. Fürnkranz. Separating rule refinement and rule selection heuristics in inductive rule learning. In T. Calders, F. Esposito, E. Hüllermeier, and R. Meo, editors, Machine Learning and Knowledge Discovery in Databases, volume 8726 of Lecture Notes in Computer Science, pages 114-129. Springer Berlin Heidelberg, 2014.

[21] T. Stützle and H. H. Hoos. Max-min ant system. Future Gener. Comput. Syst., 16(9):889-914, June 2000.

[22] I. H. Witten and E. Frank. Data Mining: Practical Machine Learning Tools and Techniques, Second Edition (Morgan Kaufmann Series in Data Management Systems). Morgan Kaufmann Publishers Inc., San Francisco, CA, USA, 2005. 